\title{
Increased macromolecular damage underlies the decreased longevity of WNIN/GR-ob rats fed high sucrose diet
}

\begin{abstract}
Ageing is a universal, multifactorial phenomenon, determined by genetic and environmental factors such as diet, stress etc. Diet associated disorders are widely prevalent in India and have been attracting much attention and research in recent times, mostly due to increased awareness among population of the importance of nutrition and balanced diet. Adaptation of western lifestyle (eg. consupmtion of calorie rich, high fat foods, lack of physical activity etc) has increased the prevalence of metabolic disorders like obesity, hypertension, Type 2 Diabetes, cardiovascular diseases etc. It is known that obesity and diabetes per se accelerate ageing, but it is not clear whether or not superimposing diabetes on obesity accelerates ageing. We tested the hypothesis that superimposing Type 2 Diabetes/aggravated insulin resistance on obesity accelerates ageing, by chronic feeding of high sucrose diet (HSD) to $\mathrm{WNIN} / \mathrm{Gr}-\mathrm{Ob}$, the obese mutant rats of NIN, which aggravated insulin resistance significantly in these obese rats. The survival analysis by Kaplan Meier plot showed decreased longevity in HSD fed rats compared to controls fed starch based diet and in line, cumulative hazard was also higher in HSD fed rats than controls. While the finding that the total DNA damage, an important ageing marker, was significantly higher in HSD fed than control rats appears to confirm the reduced longevity/accelerated ageing in them, the significantly lower telomere length in the liver and brain of HSD fed rats than their age and sex matched control rats fed starch based diet, after 6months on their respective diets appears to unequivocally demonstrate the accelerated ageing/decreased longevity in HSD fed WNIN/Gr-Ob rats. Our results support the hypothesis that aggravating insulin resistance in obese rats through high sucrose feeding decreases longevity/accelerate aging.
\end{abstract}

Keywords: high sucrose diet, type 2 diabetes, ageing, aggravated insulin resistance, WNIN/gr-ob rats, accelerated ageing, DNA damage, reduced longevity
Volume 6 Issue I - 2017

\author{
Aruna Potukuchi,' Uma Addepally, ${ }^{2}$ \\ Raghunath Manchala ${ }^{3}$ \\ 'Endocrinology and Metabolism Division, National Institute of \\ Nutrition, India \\ ${ }^{2}$ Centre for Biotechnology, IST, Jawaharlal Nehru Technological \\ University (Hyderabad), India
}

\begin{abstract}
Correspondence: Raghunath Manchala, Scientist-G and Head, Director Grade Scientist, Endocrinology and Metabolism Division, National Institute of Nutrition, Hyderabad-500007, Telangana State, India, Tel 9|4027| 97235 (O), 94406 I8I I 8; Fax 914027019074, Email mraghunath55@yahoo.com
\end{abstract}

Received: November 04, 2016 | Published: January 04, 2017
Abbreviations: HSD, high sucrose diet, SBD, starch based diet, WNIN/Gr-Ob, wistar national institute of nutrition obese rats, HOMA IR, homeostatic model assessment insulin resistance, AUC, area under curve, OGTT, oral glucose tolerance test, ROS, reactive oxygen species.

\section{Introduction}

Ageing is a universal phenomenon, defined as the process of gradual and spontaneous change, resulting in maturation through childhood, puberty, young adulthood and then decline through middle and late age. It is also the accumulation of various changes in physical, psychological, and social behavior of a person. The ever changing urbanization and life style is demanding more work, leading to stress and changed eating habits of people around the world. India is not an exception. According to WHO estimates, more than $1 \cdot 3$ billion adults worldwide are overweight, and a further 600 million are obese. ${ }^{1}$ Obesity is the major cause of disability and premature deaths in developing countries. Insulin resistance is the major etiological factor for diabetes and the risk association between obesity and diabetes is mediated through insulin resistance. ${ }^{2}$ Indeed, insulin resistance and visceral fat accumulation are two important culprits in the pathogenesis of metabolic disease and they predispose organisms to premature ageing. ${ }^{3}$
India is ranked the highest in the world for obesity induced diabetes. It is estimated by International diabetes federation that the number of people in India with diabetes is $3.9 \%$ of total population and this may rise to $5.3 \%$ by 2030 . This condition is partly due to genetic factors, specially the Asian Indian phenotype with the underlying biochemical abnormalities and partly due to dietary patterns and life style habits ${ }^{4}$ with an increased number of young people succumbing to diabetes. The alarming rise in the number of people with type II diabetes necessitates urgent attention as many of them show preaging or advanced or accelerated aging symptoms even at the age of $35-40 y e a r s$. Accelerated aging is a faster-than-normal deterioration in the functioning of the body and mind. It is fast becoming an epidemic in developed and developing countries.

Obesity is the epidemic of the 21 st century. In developing countries, not only is the prevalence of obesity continues to rise, but also obesity is occurring at younger ages. The World Health Organization estimates that globally there are $>1$ billion overweight adults [body mass index (BMI; in $\left.\mathrm{kg} / \mathrm{m}^{2}\right)>27$ ], 300million of whom are obese (BMI $\left.>30\right)$ .Obesity per se accelerates the ageing of liver, ${ }^{5}$ thymus. ${ }^{6}$ Ahima et al., ${ }^{3}$ reported that obesity accelerates the ageing of adipose tissue and in turn stated that this could be the connecting link between obesity, ageing and Type 2 Diabetes. Further, obesity is associated with increased brain age. ${ }^{7}$ In addition, Type 2 Diabetes per se accelerates 
ageing both in humans and rodents. ${ }^{8-11}$ Concomitantly Dietary habits and diet composition is a crucial player in pathophysiology of T2D. ${ }^{12}$ Consumption of high sucrose diet leads to the development of obesity, insulin resistance, Type 2 Diabetes both in rodents and humans. ${ }^{13,14}$ Moreover, intake of high energy nutrients like sugars and fatty acids interact directly with genomic expressions, termed as nutrigenomics, ultimately leading to the metabolic syndrome. ${ }^{15}$

It is clear from the above literature, that obesity and Diabetes per se can lead to ageing, but it is not clear whether superimposing Diabetes on obesity accelerates ageing and if yes the possible, underlying mechanisms. To understand the adverse effects caused by superimposing diabetes on obesity, WNIN/GR-Ob rats were used as the rodent models for obesity. These mutant rats were developed from the parental WNIN inbred rat strain maintained at the NCLAS, NIN, and Hyderabad. WNIN/GR-Ob mutants are hyper-cholesterolemic, hyper-triglyceridemic and show obesity at 35days of age. They are normoglycemic under fasting condition; are hyper-glycaemic when challenged with glucose orally ${ }^{16}$ and they exhibit impaired glucose tolerance. Considering the exceptional feature that WNIN/GR-Ob rats obese and glucose intolerant, we considered it appropriate to induce Type 2 Diabetes in these rats by feeding High Sucrose Diet (HSD) for a period of 6 months. Studies were carried out in different tissues (brain, liver) at two different time points (3 and 6months) of feeding, to correlate aging with diabetes and obesity as compared to their corresponding age and sex matched counterparts fed Starch Based Diet (SBD).

\section{Results and discussion}

Ageing is an inevitable and universal biological phenomenon It occurs at its own rate, by passing through several stages of life. Healthy Life span is influenced by several environmental factors, genetic predisposition, lifestyle related changes etc. Longevity is reduced by the progressive accumulation of macromolecular damage. Increased macromolecular damage is the strongest causative factor of ageing. In the present study the damage to macromolecules was studied in brain and liver. To the best of our knowledge this is the first study linking diet, diabetes, obesity and reduced longevity.

\section{Survival analysis to determine the longevity of HSD fed WNIN/Gr-Ob rats}

The effect if any of the HSD feeding on the longevity of rats was evaluated by Kaplan Meier survival plots and cumulative hazard plots. ${ }^{17}$ It was interesting that the survival of rats fed HSD was lower than those fed SBD indicating their reduced longevity. In line with reduced longevity, HSD fed rats had higher levels of cumulative hazards than SBD rats suggesting that the decreased longevity/ survival of the HSD fed rats could be due to higher cumulative hazard in them.

\section{Biochemical parameters to assess induction of Diabetes}

Pre-diabetic state is a pathological condition characterized by aggravated insulin resistance. ${ }^{18}$ Insulin resistance is assessed by computing HOMA IR using the fasting plasma glucose and insulin levels, in addition to determining insulin AUC during the OGTT. We observed that HSD fed rats had significantly higher fasting plasma insulin, HOMA IR and insulin AUC during the OGTT compared to their SBD counterparts, at both time points of feeding studied. However plasma glucose was significantly higher in HSD fed rats only at 3 but not 6 months of feeding. These results suggest that HSD feeding aggravated insulin resistance/induced Type 2 Diabetes/in the WNIN/Gr-Ob rats.

\section{Ageing of the Brain}

Brain is the master controller of the body which controls the functions of all other organs and Age is a major risk factor for most common neurodegenerative diseases. The functions of different regions in the brain are differentially susceptible to age-induced changes. ${ }^{19}$ Indeed, brain ageing can be considered as the death of the functioning brain and indirectly that of the body. Brain ageing is normally expressed in terms of DNA damage which is quantitated by the Comet assay. ${ }^{20}$ It was interesting that the total neuronal DNA damage was significantly higher in HSD fed rats than those fed SBD after 3 months of feeding. It was interesting that telomere length, a key marker of ageing, was significantly reduced in HSD fed rats compared to SBD fed rats, but only after 6 months of feeding. Indeed our observations that HSD fed rats had increased oxidative stress and decreased antioxidant defense in their brain are in line with our hypothesis and appear to justify the inference that High sucrose feeding to obese rats aggravates DNA damage by increasing their oxidative stress. Overall, these findings suggest that HSD feeding to WNIN/Gr-Ob rats reduces their longevity compared to those fed SBD and increased oxidative stress and the attendant DNA damage may underly this decreased longevity.

\section{Ageing of Liver}

Liver is an important metabolically active organ, whose cells turnover rapidly. The rate of ageing of the liver can be determined either by the increased DNA damage and/or decreased repair activity. Morphological and histological changes, ROS are all involved in the ageing of liver. ${ }^{21}$ Histologically, increased hepatic steatosis was observed in WNIN/Gr-Ob rats fed HSD at both the time points of feeding studied, compared to their counterparts fed SBD. Increased macromolecular damage was observed in these livers as evident from the increase in lipid peroxidation and protein carbonyl levels observed and a significant decrease in their antioxidant status, the defense mechanism that is essential to cope up with the increased oxidative stress. In addition, there was impaired DNA repair activity in these livers as evident from the significant decrease in the gap repair activity determined using synthetic labeled duplex DN. ${ }^{22}$ Since reduced telomere length is considered to be the direct evidence linking obesity and accelerated ageing, ${ }^{23}$ the significant decrease observed in the telomere length of liver DNA from HSD fed rats compared to those fed SBD is in line with and confirms the reduced longevity of the HSD fed rats as determined by the Kaplan Meier plots earlier.

\section{Telomere Length}

In mammals telomere is the terminal cap of the cells and it protects the cells from degradation. Telomere length is the well established biomarker of ageing. The length of the telomere varies with age. But in diseased conditions and in metabolic syndrome, the telomere length is affected. Considering this fact, we measured telomere length in brain, liver and neuronal cells of WNIN/Gr-Ob rats fed HSD and SBD fed control rats after 3 and 6months of feeding their respective diets (Figure 1). We observed that there were differences between organs in the lengths of telomere and the telomere length was significantly reduced with age in the brain and liver of WNIN/Gr-Ob rats fed HSD after feeding for 6months than those fed SBD. The observed is reduction in telomere length appears to support the inference that increased total neuronal DNA damage may underlie the reduced lifespan of HSD fed WNIN/Gr-Ob rats. 


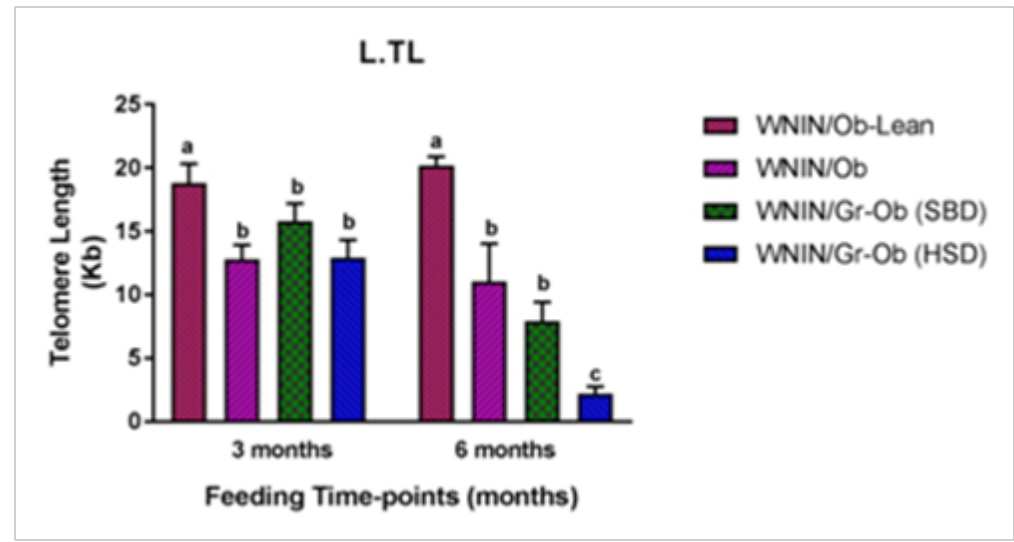

A. Telomere lengths in Liver

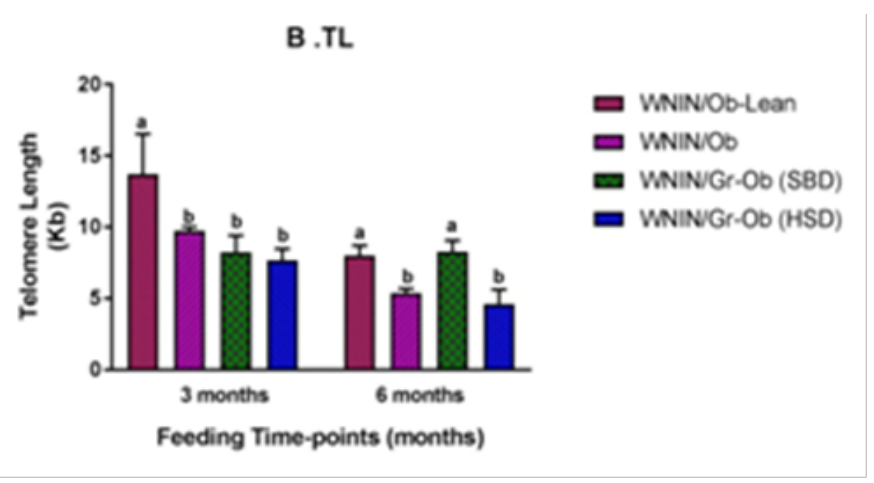

B. Telomere lengths in brain

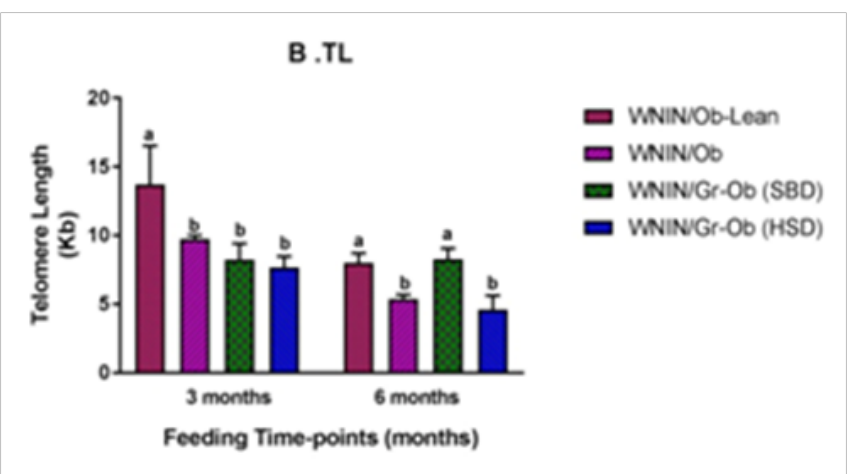

C. Telomere lengths in Isolated Neuronal cells

Figure I Telomere lengths in different groups of rats after 3 and 6 months of feeding their respective diets.

\section{Conclusion}

From our observations we conclude that inducing T2D/aggravating insulin resistance in the WNIN/Gr-Ob obese rats by high sucrose feeding reduced their longevity. Increased macromolecular (lipids, protein and DNA) damage most probably due to increased oxidative stress and/or impaired antioxidant status appear to be the underlying/ associated mechanism.

\section{Acknowledgements}

We acknowledge DBT for funding the project (BT/PR 3404/ FNS/20/529/2011), NIN/NCLAS (ICMR) for the supply of animals.

\section{Conflict of interest}

Author declares that there is no conflict of interest.

\section{References}

1. WHO. Obesity and overweight. Fact sheet $N^{\circ} 311$. Geneva: World Health Organization; 2014.

2. Ramachandran A, Snehalatha C. Rising burden of obesity in Asia. $J$ Obes. 2010;pii:868573.

3. Ahima RS. Connecting obesity, aging and diabetes. Nat Med. 2009;15(9):996-997.

4. Mohan V, Sandeep S, Deepa R, et al. Epidemiology of type 2 diabetes: Indian scenario. Indian J Med Res Mar. 2007;125(3):217-230.
5. Horvath S, Erhart W, Brosch M, et al. Obesity accelerates epigenetic aging of human liver. Proc Natl Acad Sci U S A. 2014;111(43):15538-15543.

6. Yang H, Youm YH, Vandanmagsar B, et al. Obesity accelerates thymic aging. Blood. 2009;114(18):3803-3812.

7. Ronan L, Alexander-Bloch AF, Wagstyl K, et al. Obesity associated with increased brain age from midlife. Neurobiol Aging. 2016;47:63-70.

8. Mehla J, Chauhan BC, Chauhan NB. Experimental induction of type 2 diabetes in aging-accelerated mice triggered Alzheimer-like pathology and memory deficits. J Alzheimers Dis. 2014;39(1):145-162.

9. Barzilai N, Huffman DM, Muzumdar RH, et al. The critical role of metabolic pathways in aging. Diabetes. 2012;61(6):1315-1322.

10. Palmer AK, Tchkonia T, LeBrasseur NK, et al. Cellular Senescence in Type 2 Diabetes: A Therapeutic Opportunity. Diabetes. 2015;64(7):2289-2298.

11. Gong Z, Muzumdar RH. Pancreatic function, type 2 diabetes, and metabolism in aging. Int $J$ Endocrinol. 2012;2012:320482.

12. Buyken AE, Mitchell P, Ceriello A, et al. Optimal dietary approaches for prevention of type 2 diabetes: a life-course perspective. Diabetologia. 2010;53(3):406-418.

13. Bizeau ME, Pagliassotti MJ. Hepatic adaptations to sucrose and fructose. Metabolism. 2005;54(9):1189-1201.

14. Sandra BS, Isabele BS, Thereza C, et al. Animal Models of Nutritional Induction of Type 2 Diabetes Mellitus. Int J Morphol. 2014;32(1):279-293. 
15. Minihane AM. Nutrient gene interactions in lipid metabolism. Curr Opin Clin Nutr Metab Care. 2009;12(4):357-363.

16. Harishankar N, Vajreswari A, Giridharan NV. WNIN/GR-Ob - an insulin-resistant obese rat model from inbred WNIN strain. Indian $J$ Med Res. 2011;134:320-329.

17. Yang JS, Nam HJ, Seo M, et al. OASIS: online application for the survival analysis of lifespan assays performed in aging research. PLoS One. 2011;6(8):e23525.

18. Davidson MB. Metabolic syndrome/insulin resistance syndrome/prediabetes: new section in diabetes care. Diabetes Care. 2003;26(11):3179.

19. Giorgio A, Santelli L, Tomassini V, et al. Age-related changes in grey and white matter structure throughout adulthood. Neuroimage. 2010;51(3):943-951.
20. Swain U, Subba Rao K. Study of DNA damage via the comet assay and base excision repair activities in rat brain neurons and astrocytes during aging. Mech Aging Dev. 2011;132(8-9):374-381.

21. Anantharaju A, Feller A, Chedid A. Aging Liver. A review. Gerontology. 2002;48(6):343-353.

22. Krishna TH, Mahipal S, Sudhakar A, et al. Reduced DNA gap repair in aging rat neuronal extracts and its restoration by DNA polymerase beta and DNA-ligase. J Neurochem. 2005;92(4):818-823.

23. Tzanetakou IP, Katsilambros NL, Benetos A, et al. "Is obesity linked to aging?": adipose tissue and the role of telomeres. Ageing Res Rev. 2012;11(2):220-229. 